\title{
An Application of Cyclotomic Polynomial to Factorization of Finite Abelian Groups*
}

\author{
Khalid Amin \\ University of Bahrain, Department of Mathematics, Sakhir, Kingdom of Bahrain \\ E-mail:kamin@sci.uob.bh \\ Received June 21, 2011; revised July 25, 2011; accepted August 21, 2011
}

\begin{abstract}
A finite abelian group $G$ is said to have the Hajós-k-property $(\mathrm{k}>1)$ if from any decomposition $G=A_{1} A_{2} \ldots A_{K}$ of $G$ into a direct product of its subsets, it follows that one of these subsets $A_{i}$ is periodic, meaning that there exists a nonidentity element $g$ in $G$ such that $g A_{i}=A_{i}$. Using some properties of the cyclotomic polynomials, we will show that the cyclic groups of orders $p^{\alpha}$ and groups of type $\left(p^{2}, q^{2}\right)$, where $p$ and $q$ are primes have this property. We also include a partial result about groups of type $\left(p^{\alpha}, q^{\beta}\right)$, where $p$ and $q$ are distinct primes and $\alpha, \beta$ are integers $\geq 1$.
\end{abstract}

Keywords: Factorization of Finite Abelian Groups, Hajós Property

\section{Introduction}

Let $G$ be a finite abelian group with identity element $e$. If $G$ is a direct product of cyclic groups of orders $m_{1}, m_{2}, \cdots, m_{r}$, we say that $G$ is of type $\left(m_{1}, m_{2}, \cdots, m_{r}\right)$. If $A_{1}, A_{2}, \cdots, A_{k}$ are subsets of $G$ such that each element $g$ of $G$ can be expressed in a unique way as $g=a_{1} a_{2} \cdots a_{k}$, where $a_{i} \in A_{i}$, we write $G=A_{1} A_{2}, \cdots A_{k}$ and say that we have a factorization of $G$. If in addition each contains the identity element $G$, we say that we have a normalized factorization of $G$. We will use $\left|A_{i}\right|$ to denote the number of elements of $A_{i}$. Similarly $|g|$ will donate the order of the element $g$ of $G$. A subset $A$ of $G$ is called periodic if there is a non-identity element $g$ in $G$ such that $g A=A$.

The topic of factorizations of abelian groups arose when Hajós [2] solved a conjecture by H. Minkowski [3] concerning lattice tiling after transforming it into a theorem about finite abelian groups. For reference, we state this theorm below:

If $G=A_{1} A_{2}, \cdots A_{k}$ is a factorization of a finite abelian group $G$, where each of the subsests is of the form $\left\{e, a, a, \cdots a^{r}\right\}$, then at least one of these subsets is a subgroup of $G$.

L. Rédei [4] generalized this to:

If $G=A_{1} A_{2}, \cdots A_{k}$ is a factorization of a finite abe-

"Mathematics Subject Classification: 20K01 lian group $G$, where each of the subsets has a prime number of elements and contains the identity $e$, then at least one of these subsets is a subgroup of $G$.

A. Sands [5] classified groups with Hajós-2-proprty which we list below:

$$
\begin{gathered}
\left(p^{\alpha}, q\right),\left(p^{2}, q^{2}\right),\left(p^{2}, q, r\right),(p, q, r, s) \\
\left(p^{3}, 2,2\right),\left(p^{2}, 2,2,2\right),\left(p, 2^{2}, 2\right),(p, 2,2,2,2) \\
(p, q, 2,2),(p, 3,3),\left(3^{2}, 3\right),\left(2^{\alpha}, 2\right) \\
\left(2^{2}, 2^{2}\right),(p, p)
\end{gathered}
$$

where $p, q, r$ and $s$ are primes and $\alpha \geq 1$ is an integer.

\section{Preliminaries}

Let $G$ be a cyclic group of order $n$, with generator $g$. Let us write $G=\sum_{i=0}^{n-1} g^{i}$. Similarly, for a subset $A=\left\{g^{\alpha_{1}}, g^{\alpha_{2}}, \cdots, g^{\alpha_{r}}\right\}$ of $G$, we write $A=\sum_{i=0}^{r} g^{\alpha_{i}}$.

Then we can write

$$
A_{1} A_{2} \cdots A_{k}=\left(\sum_{i=1}^{\eta_{1}} g^{\alpha_{i}}\right)\left(\sum_{i=1}^{r_{2}} g^{\alpha_{i}}\right) \cdots\left(\sum_{i=1}^{r_{2}} g^{\alpha_{i}}\right),
$$

Where multiplication is carried out in the group ring $Z(G)$. Thus, when multiplication is carried out we regard $A_{i}$ as polynomials in $g$ provided that addition of 
the indices is carried out module $n$. i.e. polynomials are multiplied $\bmod \left(g^{n}-1\right)$.

Now, if we replace $g$ by $x$ and write $A_{i}(x)=\sum_{i=1}^{r_{i}} x^{\alpha_{i}}$ then from the relation $G=A_{1} A_{2}, \cdots A_{k}$, then we get:

$$
G(x)=A_{1}(x) A_{2}(x) \ldots A_{k}(x) \bmod \left(x^{n}-1\right)
$$

As $\left(x^{n-1}+\cdots x+1\right)$ is a factor of $x^{n}-1$, it follows that each irreducible divisor of $\left(x^{n-1}+\cdots x+1\right)$ will divide one of the polynomials $A_{i}(x)$. These irreducible polynomials are the cyclotomic polynomials whose roots are the $d$-th primitive roots of unity where $d \mid n$ and $d>1$.

At some stage in this work, we shall need the following facts about the cyclotomic polynomials.

1) The $n$-th cyclotomic polynomial is usually denoted by $\Phi_{n}(x)$ and is given by:

$$
\Phi_{n}(x)=\prod_{d \mid n}\left(1-x^{\frac{n}{d}}\right)^{\mu(d)}
$$

2) The $\Phi_{n}(x)^{\prime} s$ have integer coefficients i.e. $\Phi_{n}(x)$ $\in Z(x)$ and they are irreducible and relatively prime.

Slightly modifying the notation of De Bruijn [1], we also define for a divisor $d$ of $n$, the polynomial

$$
\Phi_{n, d}(x)=\frac{1-x^{n}}{1-x^{\frac{n}{d}}}=1+x^{\frac{n}{d}}+x^{2 \frac{n}{d}}+\cdots+x^{(d-1) \frac{n}{d}}
$$

\section{Results}

Before we embark on showing our results, we must mention that all factorizations can be assumed to be normalized, for if $G=A_{1} A_{2}, \cdots A_{k}$ is a factorization of $G$, then since each $A_{i}$ is non-empty, there exists an element $a_{i}$ is $A_{i}, 1 \leq i \leq k$. Multiplying $G$ by $g=\left(a_{1} a_{2}, \cdots a_{k}\right)^{-1}$, we get that $G=g G=a_{1}^{-1} A_{1} a_{2}^{-1} A_{2}, \cdots a_{k}^{-1} A_{k}$, which is clearly normalized.

Theorem 3.1 Let $p$ be a prime. If $G$ is a cyclic group of order $n=p^{\alpha}$, then $G$ has the Hajos- $k$-proprty, for all $k, 1<k \leq \alpha$.

Proof Let $G$ be generated by $g$ and consider the factorization $G=A_{1} A_{2}, \cdots A_{k}$ of $G$. Our previous discussion leads to the following congruence relation:

$$
G(x) \equiv A_{1}(x) A_{2}(x) \cdots A_{k}(x) \bmod \left(x^{n}-1\right) .
$$

Now, $\Phi_{n}(x)$ divides some $A_{i}(x)$. But

$$
\Phi_{n}(x)=\Phi_{p^{\alpha}}(x)=\frac{x^{p^{\alpha}}-1}{x^{p^{\alpha-1}}-1} .
$$

Thus,

$\frac{x^{p^{\alpha}}-1}{x^{p^{\alpha-1}}-1}$ divides $A_{i}(x)$. It follows that $A_{i}$ is periodic with period $g^{p^{\alpha-1}}$

As an illustration, consider a cyclic group $G=\langle g\rangle$ of order $8=2^{3}$. Let $A=\left\{e, g, g^{4}, g^{5}\right\}$ and $B=\left\{e, g^{2}\right\}$. Then it is easy to verify that $A B=G$ is a factorization of $G$ and that $A$ is periodic with period $g^{4}=g^{2^{3-1}}$.

We shall use the following theorem by De Bruijn [1] in showing our next result.

If $n=p^{\alpha} q^{\beta}$, where $p, q$ are distinct primes and $\alpha, \beta \geq 1, F(x) \in Z[x]$ and $\Phi_{n}(x)$ divides $F(x)$, then $F(x)=g(x) \Phi_{n, p}(x)+h(x) \Phi_{n, q}(x)$ for some polynomials $g(x), \quad h(x) \in Z[x]$.

Theorem 3.2 If $G$ is of type $\left(p^{2}, q^{2}\right)$, where $p$ and $q$ are distinct primes, then $G$ has the Hajos-k-property, for all $k, 1<k \leq 4$.

Proof Consider a factorization $G=A_{1} A_{2}, \cdots A_{k}$ of $G$. The case $k=4$ is true by Redei's theorem. The case $k=3$, is true by Redei theorem and Theorem 2 of Sands [5]. Thus, we only need detail the case $k=2$. Say $G=A_{1} A_{2}$ in which both factors contain $p q$ elements. Again by our previous discussion, we obtain the relation

$$
G(x) \equiv A_{1}(x) A_{2}(x) \bmod \left(x^{n}-1\right),
$$

where $n=p^{2} q^{2}$. It follows that $\Phi_{n}(x)$ divides $A_{1}(x) A_{2}(x)$. Since $A_{1}$ and $A_{2}$ contain the same number of elements, we may and shall assume that $\Phi_{n}(x)$ divides $A_{1}(x)$. Then, by De Bruijn's result above, we get that

$$
A_{1}(x)=f(x) \Phi_{n, p}(x)+g(x) \Phi_{n, q}(x) .
$$

Therefore,

Now:

$$
A_{1}(x)=f(x) \frac{x^{n}-1}{x^{\frac{n}{p}}-1}+g(x) \frac{x^{n}-1}{x^{\frac{n}{q}}-1} .
$$

$$
A_{1}(1)=p q=p f(1)+q g(1)
$$

Therefore, either $f(1)=q$ and $g(1)=0$, or $f(1)=$ 0 and $g(1)=p$. In the first case, $g(x)=0$, and $\frac{x^{n}-1}{x^{n / p}-1}$ divides $A_{1}(x)$ in which case $A_{1}$ is periodic with period $g^{n / p}$. In the second case, $f(x)=0$ and $\frac{x^{n}-1}{x^{n / q}-1} \operatorname{divides} A_{1}(x)$ and so $A_{1}$ is periodic with period $g^{n / p}$.

Corollary 3.1 If $G$ is of type $\left(p^{\alpha}, q^{\beta}\right)$, where $p$ and $q$ are distinct primes, and $\alpha, \beta$ are integers $\geq 1$, then $G$ 
has the Hajós- $k$-property, where $k=(\alpha+\beta) / 2$.

\section{References}

[1] N. G. De Bruijn, "On the Factorization of Finite Cyclic Groups," Indagationes Mathematicae, Vol. 15, No.4, 1953, pp. 370-377.

[2] G. Hajos, "Uber Einfache und Mehrfaache Bedekung des n-Dimensionales Raumes Mit Einem Wurfelgitter," Mathematics Zeitschrift, Vol. 47, No. 1, 1942, pp. 427-467.

\section{doi:10.1007/BF01180974}

[3] H. Minkowski, "Diophantische Approximationen," Teuner, Leipzig, 1907.

[4] L. Redei, "Ein Beitrag Zum Problem Der Faktorisation Von Endlichen Abelschen Gruppen," Acta Mathematics Hungarica, Vol. 1, No. 2-4, 1950, pp. 197-207. doi:10.1007/BF02021312

[5] A. Sands, "Factorization of Finite Abelian Groups," Acta Mathematics Hungarica, Vol. 13, No. 1-2, 1962, pp. 153169. doi:10.1007/BF02033634 